\title{
Linear least squares localization in sensor networks
}

Yue Wang

\begin{abstract}
Localization in sensor networks is critical for search and rescue. Linear least squares (LLS) estimation is a sub-optimum but low-complexity localization algorithm based on measurements of location-related parameters. Commonly, there are two types of LLS localization algorithms using range measurements; one is based on introducing a dummy variable (called LLS-I), and the other is based on the subtraction of the reference measured range (called LLS-I). Moreover, their respective weighted LLS (WLLS) algorithms (called WLLS-I and WLLS-II) can be adopted to further improve the localization accuracy. In addition, hybridization of different types of measurements can fix the deficiencies of one type of measurements. In this paper, we compare the localization performances of different LLS and WLLS algorithms in both non-hybrid time-of-arrival (TOA) and hybrid TOA/received signal-strength (RSS) networks. Simulation results show that if the variances of measurements are unavailable, the LLS-II localization algorithm should be adopted in both non-hybrid and hybrid networks using their respective reference selection criterions. If the variances of measurements are available, the two-step WLLS-I algorithm should be utilized to localize the agent in both non-hybrid and hybrid networks.
\end{abstract}

Keywords: Hybrid localization; Received-signal-strength (RSS); Reference selection; Time-of-arrival (TOA); Ultra-wideband (UWB); Weighted linear least squares (WLLS)

\section{Introduction}

Determining the location of a target is one of the fundamental functions of sensor networks [1-5]. Real-time and accurate position information plays a crucial role in a variety of wireless applications [6,7], such as logistics, searchand-rescue operations, and location-based services. In the conventional two-step localization method [8], locationrelated parameters, such as range and angle-of-arrival (AOA) information, are estimated in the first step, and the position of the agent is then estimated based on these location-related parameters using geometric and statistical algorithms. Specifically, range information is commonly adopted in the first step of localization, which can be measured using time-of-arrival (TOA) or received-signalstrength (RSS) estimates [9]. Maximum likelihood (ML) estimator can be adopted in the second step of rangebased localization if the variances of range measurements are available. It is well known that ML estimator is an optimum estimator since it can asymptotically achieve the Cramér-Rao lower bound (CRLB) for high signal-to-

Correspondence: ywang_tinu@163.com

College of Electronic and Communication Engineering, Tianjin Normal University, Tianjin 300387, China noise ratios (SNRs) and/or large signal bandwidths [9]. If the variance information about measured range is unavailable, a nonlinear least squares (NLS) estimator can be adopted, which assumes all the variances are identical and employs uniform weighting. Solving the NLS estimation problem requires an explicit minimization of a nonlinear as well as non-convex cost function, which cannot in general be solved analytically. Therefore, numerical search algorithms, such as the Newton-Newton, the Gauss-Raphson, or the steepest descent algorithms, are usually employed to approximate the NLS estimate [6]. However, the drawbacks of these numerical search algorithms are intensive computation as well as good initialization requirement to avoid erroneously converging to the local minima of the NLS cost function [7].

In order to avoid the explicit minimization problem and obtain a closed form solution, the nonlinear expressions of observations can be linearized using the linear least squares (LLS) algorithms. Commonly, there are two types of LLS algorithms using range measurements. One type of LLS algorithm is based on the utilization of a dummy variable (called $L L S-I$ ) [10]. The other type of LLS algorithm is based on the subtraction of the reference

\section{(1)}


measured range (called $L L S-I I$ ), which was initially proposed in [11] and its reference selection method was discussed in [12] and [13] for non-hybrid networks and hybrid TOA/RSS networks, respectively. For the line-ofsight (LOS) and non-line-of-sight (NLOS) application scenarios, the LLS localization performance was studied in $[12,14]$. Compared with iterative algorithms (e.g., ML and NLS approaches), LLS estimation is a sub-optimum localization algorithm [15]. However, LLS estimation usually has a reasonable positioning accuracy and lower implementation complexity, which is essential to many wireless applications, such as internet of things and wireless sensor networks, due to their strict constraints on energy consumption, signal processing capability, cost, and so on. Moreover, the weighted LLS (WLLS) algorithms can further improve the LLS localization accuracy. The WLLS localization algorithms based on the utilization of a dummy variable (called WLLS- $I$ ) were discussed in [16-18], where the two-step WLLS-I localization technique outperforms the conventional one-step WLLS-I localization technique due to that the constraint of the dummy variable is exploited, while the other one based on the subtraction of the reference measured range (called WLLS-II) was discussed in $[12,13]$.

Hybrid localization techniques $[19,20]$, such as the hybrid TOA/RSS or AOA/RSS techniques, are proposed to enhance localization accuracy as well as fix the deficiencies of one type of measurements, such as the insufficiency of measurements to uniquely localize the agent. The effect of inaccurate measurements on hybrid AOA/RSS LLS localization was discussed in [21].

In this paper, we compare the localization performances of different LLS and WLLS algorithms in both non-hybrid TOA and hybrid TOA/RSS networks, which gives guidance to localization algorithm selection in different sensor networks. Simulation results show that if the variances of measurements are unavailable, the LLS-II localization algorithm should be adopted in both non-hybrid and hybrid networks using their respective reference selection criterions. If the variances of measurements are available, the two-step WLLS-I algorithm should be utilized to localize the agent in both non-hybrid and hybrid networks.

The rest of the paper is organized as follows. Section 2 introduces the system model. Section 3 briefly reviews the two common LLS localization algorithms. The WLLS location estimators are given in Section 4. Section 5 provides simulation results, and the last section concludes the paper.

\section{System model}

For the hybrid TOA/RSS network, we assume that the agent is connected to different anchors, and these anchors are able to measure the range between the agent and anchors via TOA or RSS parameters. Let $N$ be the total number of all anchors in the localization network. Without loss of generality, we assume that the agent can measure TOA-based ranges from anchors with indexes $i \in\{1,2, \ldots, S\}$ and measure RSS-based ranges from anchors with indexes $i \in\{S+1, S+2, \ldots, N\}(S \leq N)$. For the non-hybrid TOA network, we assume that the agent can measure TOA-based ranges from all $N$ anchors, i.e., $S=N$.

In the first localization step, the range measurement between the agent and the $i$ th $(i=1,2, \ldots, N)$ anchor is denoted as $\hat{d}_{i}$. Let $\mathbf{p}=[x y]^{T}$ be the unknown two-dimensional (2-D) coordinate of the agent, which is to be estimated, and let $\mathbf{p}=\left[x_{i} y_{i}\right]^{T}$ be the known 2-D coordinate of the $i$ th anchor. The error-free range between the agent and the $i$ th anchor is calculated as

$$
d_{i}=\mathbf{p}-\mathbf{p}_{i}=\sqrt{\left(x-x_{i}\right)^{2}+\left(y-y_{i}\right)^{2}}
$$

The range measurement is modeled as

$$
\hat{d}_{i}=d_{i}+n_{i}
$$

where $n_{i}$ is the ranging error in $\hat{d}_{i}$, which results from TOA or RSS estimation disturbance. It is assumed that $\left\{n_{i}\right\}$ are independent Gaussian processes with zero mean and variances $\left\{\sigma_{i}^{2}\right\}$.

Obtaining all the range measurements in (2) leads to the following inconsistent equations

$$
\left(x-x_{i}\right)^{2}+\left(y-y_{i}\right)^{2}=\hat{d}_{i}^{2}, i=1,2, \cdots, N
$$

For TOA-based range measurements, the two-way TOA ranging protocol is adopted [9] and the range can be calculated as

$$
\hat{d}=c \cdot \frac{\hat{\tau}_{\mathrm{RTT}}-\hat{\tau}_{\mathrm{TAT}}}{2}
$$

where $c$ is the propagation speed of ranging signals, $\hat{\tau}_{\text {RTT }}$ is measured round trip time, and $\hat{\tau}_{\mathrm{TAT}}$ is measured turnaround time. In additive white Gaussian noise (AWGN) channel, the CRLB of mean square ranging error (MSRE) from the $i$ th $(i \in\{1,2, \ldots, S\})$ anchor is [8]

$$
\sigma_{i, \mathrm{CRLB}, \mathrm{TOA}}^{2}=\frac{c^{2}}{8 \pi \mathrm{SNR}_{i} \beta^{2}}
$$

where $\mathrm{SNR}_{i}=\varepsilon_{i} / \mathcal{N}_{0}$ is $\mathrm{SNR}$ from the $i$ th anchor, $\varepsilon_{i}$ denotes the received signal energy, $\mathcal{N}_{0}$ denotes the oneside power spectral density of AWGN, and $\beta$ denotes the effective signal bandwidth defined by 


$$
\beta=\left(\frac{\int_{-\infty}^{+\infty} f^{2}|K(f)|^{2} d f}{\int_{-\infty}^{+\infty}|K(f)|^{2} d f}\right)^{\frac{1}{2}}
$$

with $K(f)$ denoting the Fourier transform of the ranging signal. In harsh environments, such as in buildings or urban canyons, ultra-wideband (UWB) signals can be adopted to achieve high-accuracy range measurements [22].

For RSS-based range measurements, the log-normal model is commonly used to calculate range from path loss, which is given by

$$
\bar{P}_{r}(d)=P_{0}-10 \gamma \log _{10}\left(\frac{d}{d_{0}}\right)+V
$$

where $\bar{P}_{r}(d)$ denotes the average received power in decibels at a distance $d, P_{0}$ denotes the received power in decibels at reference distance $d_{0}, \gamma$ is the path-loss exponent with typical value between 2 and 6 , and $V$ is commonly modeled as a zero-mean Gaussian random variable with variance $\sigma_{\mathrm{sh}}^{2}$, which represents the largescale fading variations (i.e., shadowing) [9]. The CRLB of MSRE from the $i$ th $(i \in\{S+1, S+2, \ldots, N\})$ anchor is [8]

$$
\sigma_{i, \mathrm{CRLB}, \mathrm{RSS}}^{2}=\left(\frac{(\ln 10) \sigma_{\mathrm{sh}} d_{i}}{10 \gamma}\right)^{2}
$$

Although there seems a simple relation between average received power and distance, it is quite challenging to obtain the exact relation between them. Since RSS can have significant fluctuations even over short distances and/or small time intervals in a practical wireless environment due to complicated signal propagation mechanisms, therefore, the accuracy of RSS-based ranging is commonly low, which is also true for UWB signals. For example, according to the IEEE 802.15.4a NLOS residential UWB channel model [23], the lower bound $\sigma_{i, \text { CRLB,RSS }}$ is about $1.76 \mathrm{~m}$ at $d_{i}=10 \mathrm{~m}$ with $\gamma=4.58$ and $\sigma_{\mathrm{sh}}=3.51 \mathrm{~dB}[8]$.

\section{LLS localization algorithms}

In the second localization step, we adopt different LLS localization algorithms to convert the nonlinear range measurements in (3) into linear models in $\mathbf{p}$ and give a close form location estimate $\hat{\mathbf{p}}_{\text {LLS }}$.

\subsection{LLS-I localization algorithm}

By reorganizing (3), we have

$$
-2 x_{i} x-2 y_{i} y+x^{2}+y^{2}=\hat{d}_{i}^{2}-x_{i}^{2}-y_{i}^{2} .
$$

We introduce a dummy variable $R=x^{2}+y^{2}$ and define $\Lambda \triangleq\left[\begin{array}{lll}x & y & R\end{array}\right]^{T}$. Then, we can rewrite (9) in matrix form as

$$
\mathbf{A}_{\mathbf{I}} \boldsymbol{\Lambda}=\mathbf{b}_{\mathbf{I}}
$$

where

$$
\mathbf{A}_{\mathbf{I}}=\left[\begin{array}{ccc}
-2 x_{1} & -2 y_{1} & 1 \\
-2 x_{2} & -2 y_{2} & 1 \\
\vdots & \vdots & \vdots \\
-2 x_{N} & -2 y_{N} & 1
\end{array}\right], \mathbf{b}_{\mathbf{I}}=\left[\begin{array}{c}
\hat{d}_{1}^{2}-x_{1}^{2}-y_{1}^{2} \\
\hat{d}_{2}^{2}-x_{2}^{2}-y_{2}^{2} \\
\vdots \\
\hat{d}_{N}^{2}-x_{N}^{2}-y_{N}^{2}
\end{array}\right]
$$

Given the linear model in (10), the LLS estimate of the vector $\Lambda$ is given by [10]

$$
\hat{\boldsymbol{\Lambda}}_{\mathrm{LLS}}=\left(\mathbf{A}_{\mathbf{I}}^{T} \mathbf{A}_{\mathbf{I}}\right)^{-1} \mathbf{A}_{\mathbf{I}}^{T} \mathbf{b}_{\mathbf{I}}
$$

The $L L S-I$ location estimate of the agent is simply extracted from the first and second entries of $\hat{\Lambda}_{\mathrm{LLS}}$, that is

$$
\widehat{\mathbf{p}}_{\mathrm{LLS}-\mathrm{I}}=\left[\left[\widehat{\boldsymbol{\Lambda}}_{\mathrm{LLS}}\right]_{1}\left[\widehat{\boldsymbol{\Lambda}}_{\mathrm{LLS}}\right]_{2}\right] T
$$

\subsection{LLS-II localization algorithm}

We choose the $r$ th anchor as the reference anchor for example without loss of generality. By subtracting the nonlinear expression of the $r$ th anchor in (3) from the rest of the expressions, we have

$$
2\left(x_{i}-x_{r}\right) x+2\left(y_{i}-y_{r}\right) y=d_{r}^{2}-d_{i}^{2}-k_{r}+k_{i}
$$

where we define $k_{i} \triangleq x_{i}^{2}+y_{i}^{2}, i=1,2, \ldots, N$.

Rewrite (13) in matrix form, we have

$$
\mathbf{A}_{\text {II }} \mathbf{p}=\mathbf{b}_{\text {II }}
$$

where

$$
\mathbf{A}_{\mathbf{I I}}=2\left[\begin{array}{cc}
x_{1}-x_{r} & y_{1}-y_{r} \\
\vdots & \vdots \\
x_{r-1}-x_{r} & y_{r-1}-y_{r} \\
x_{r+1}-x_{r} & y_{r+1}-y_{r} \\
\vdots & \vdots \\
x_{N}-x_{r} & y_{N}-y_{r}
\end{array}\right], \mathbf{b}_{\mathbf{I I}}=\left[\begin{array}{c}
d_{r}^{2}-d_{1}^{2}-k_{r}+k_{1} \\
\vdots \\
d_{r}^{2}-d_{r-1}^{2}-k_{r}+k_{r-1} \\
d_{r}^{2}-d_{r+1}^{2}-k_{r}+k_{r+1} \\
\vdots \\
d_{r}^{2}-d_{N}^{2}-k_{r}+k_{N}
\end{array}\right] .
$$

Given the linear model in (14), the LLS-II location estimate of the agent is given by [6]

$$
\widehat{\mathbf{p}}_{\text {LLS-II }}=\left(\mathbf{A}_{\text {II }}^{T} \mathbf{A}_{\text {II }}\right)^{-1} \mathbf{A}_{\text {II }}^{T} \mathbf{b}_{\text {II }}
$$

Random selection (called LLS-II-1) is the simplest way for reference selection [11], which arbitrarily selects one anchor as the reference anchor to realize LLS localization. The second LLS approach (called LLS-II-2) obtains $N \times(N-1) / 2$ linear equations by selecting each anchor as the reference anchor for the other $N-1$ measurements [14]. In the third LLS method (called LLS-II-3), the average of all range measurements is obtained as the reference range, thus resulting in $N$ linear equations [15]. For non-hybrid localization network, the fourth LLS technique (called LLS-II-RS) selects the reference anchor with the shortest measured range 


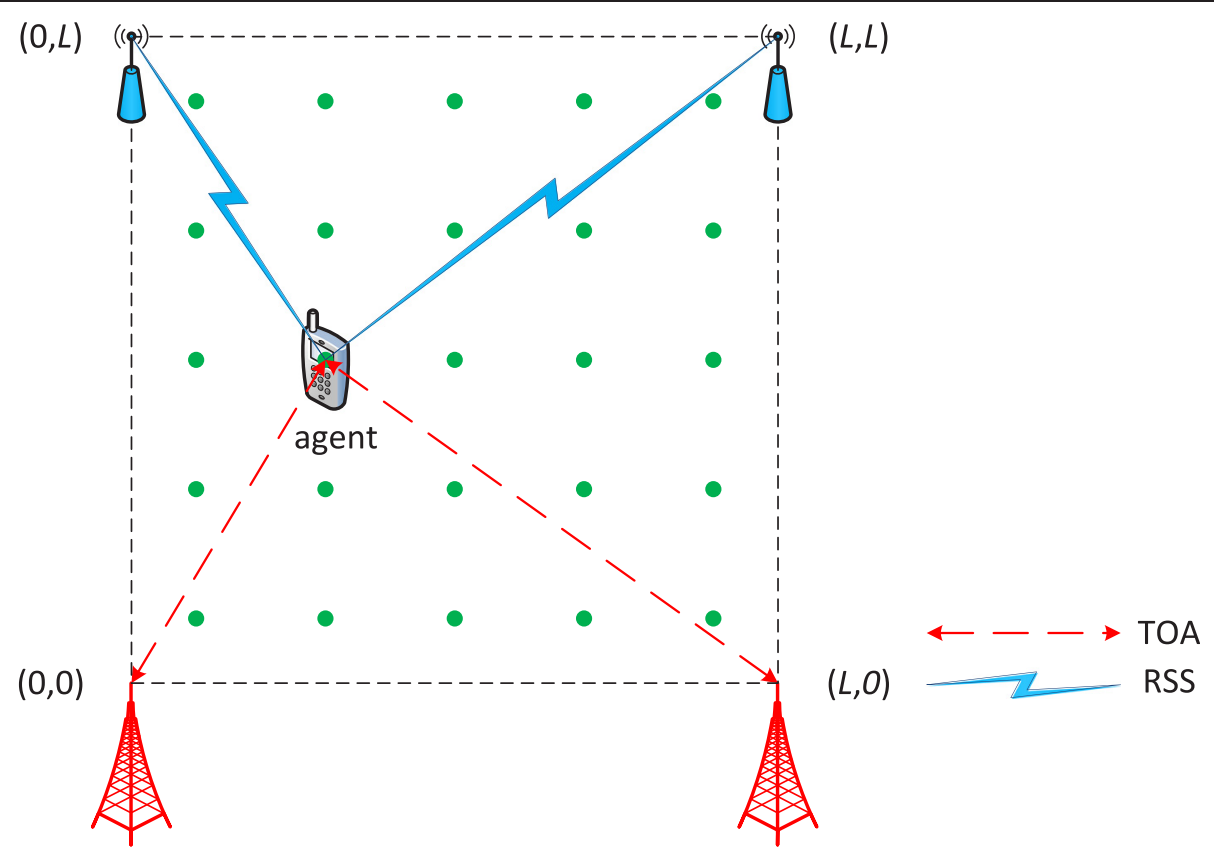

Figure 1 Hybrid TOA/RSS LLS localization network.

among all the range measurements [12], and the index of the reference anchor is given by

$$
r=\arg \min _{i}\left\{\hat{d}_{i}\right\}, i=1,2, \cdots, N
$$

For hybrid TOA/RSS localization network, if coarse information of ranging variances is available, e.g., the RSS-based ranging variances are considerably larger than the TOA-based ranging variances, the fifth LLS technique (called $H$-LLS-II-RS) selects the TOA-based anchor with the shortest measured range as reference [13], and the index of the reference anchor is given by

$$
r=\arg \min _{i}\left\{\hat{d}_{i}\right\}, i \in \mathcal{C}_{\mathrm{TOA}}
$$

where $C_{\mathrm{TOA}}$ denotes the index set for all the TOAbased anchors.

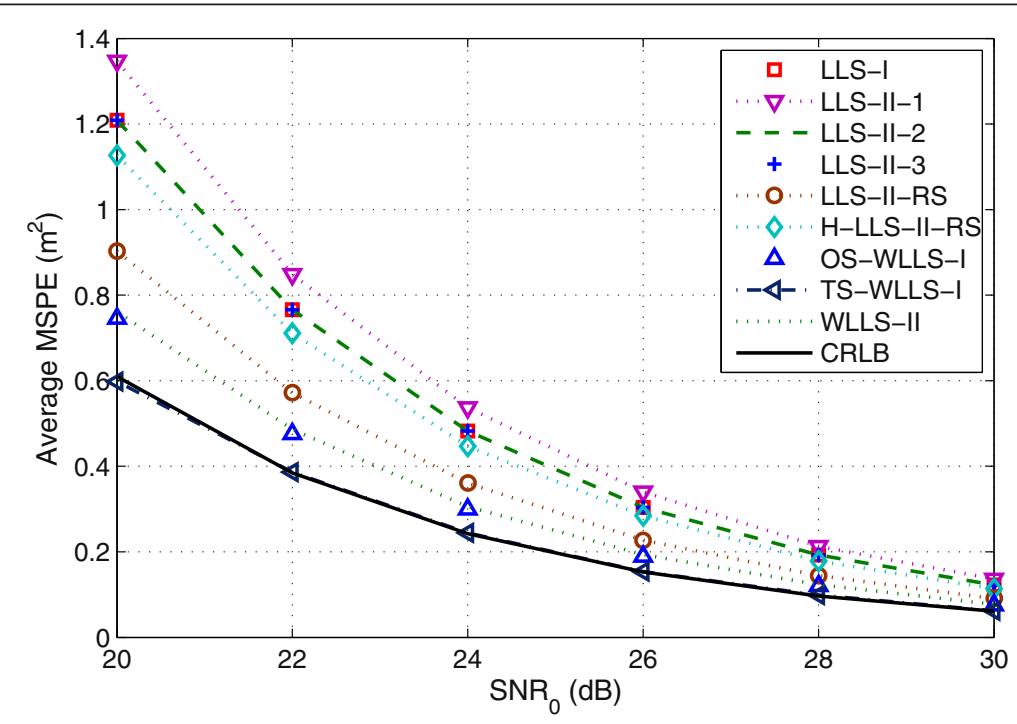

Figure 2 Comparison of different LLS localization algorithms $(\eta=1)$. 


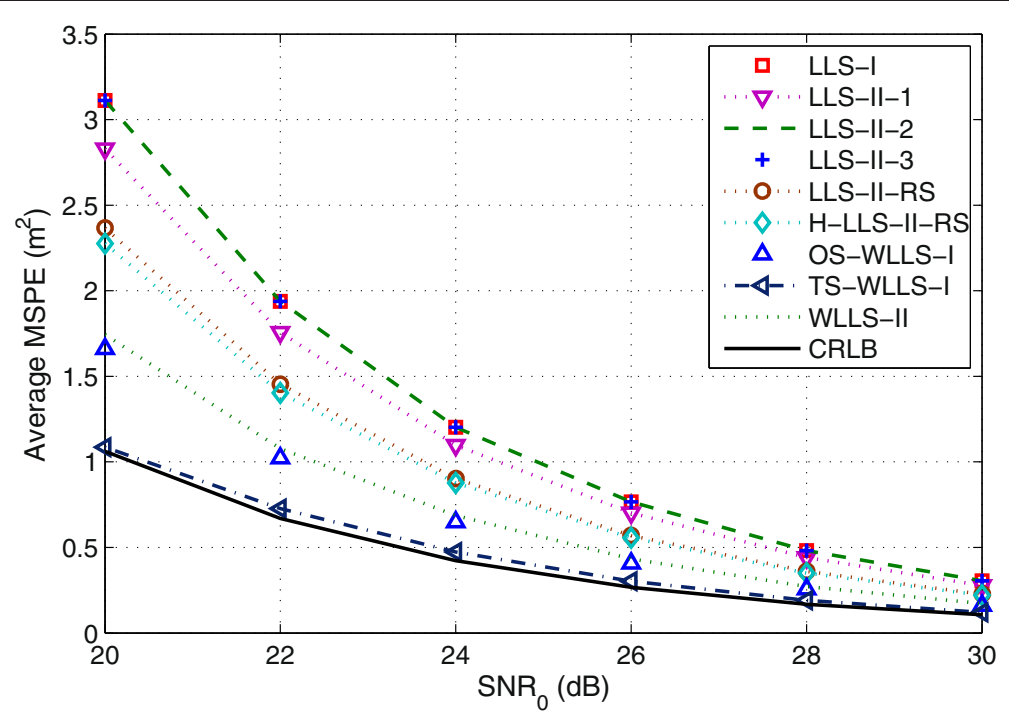

Figure 3 Comparison of different LLS localization algorithms $(\eta=2)$.

\section{WLLS localization algorithms}

If the variances of measurements are available, the WLLS localization algorithms can be adopted to further improve the localization accuracy compared with LLS localization algorithms discussed above.

\subsection{WLLS-I localization algorithm}

According to [6], the one-step WLLS-I location estimate (called OS-WLLS-I) can be written as

$$
\hat{\mathbf{p}}_{\text {OS-WLLS-I }}=\left[\left[\widehat{\Lambda}_{\mathrm{WLLS}}\right] 1\left[\widehat{\Lambda}_{\mathrm{WLLS}}\right] 2\right] T
$$

where

$$
\begin{aligned}
\widehat{\boldsymbol{\Lambda}}_{\mathrm{WLLS}} & =\left(\mathbf{A}_{\mathbf{I}}^{T} \mathbf{C}_{\mathbf{I}}^{-1} \mathbf{A}_{\mathbf{I}}\right)^{-1} \mathbf{A}_{\mathbf{I}}^{T} \mathbf{C}_{\mathbf{I}}^{-1} \mathbf{b}_{\mathbf{I}} \\
\mathbf{C}_{\mathbf{I}} & =4 \times \operatorname{diag}\left(\sigma_{1}^{2} d_{1}^{2}, \sigma_{2}^{2} d_{2}^{2}, \cdots, \sigma_{N}^{2} d_{N}^{2}\right) .
\end{aligned}
$$

Symbol diag(a) denotes a diagonal matrix with its main diagonal entries being vector a. Note that since the errorfree ranges $\left\{d_{i}\right\}$ are not available in practice, the noisy measurements $\left\{\hat{d}_{i}\right\}$ are used to evaluate the weighted matrix $\mathrm{C}_{\mathrm{I}}$.

Moreover, the two-step WLLS-I localization algorithm (called TS-WLLS-I) utilizes the constraint of the dummy variable $R=x^{2}+y^{2}$ to further enhance the localization accuracy compared with the OS-WLLS-I algorithm.

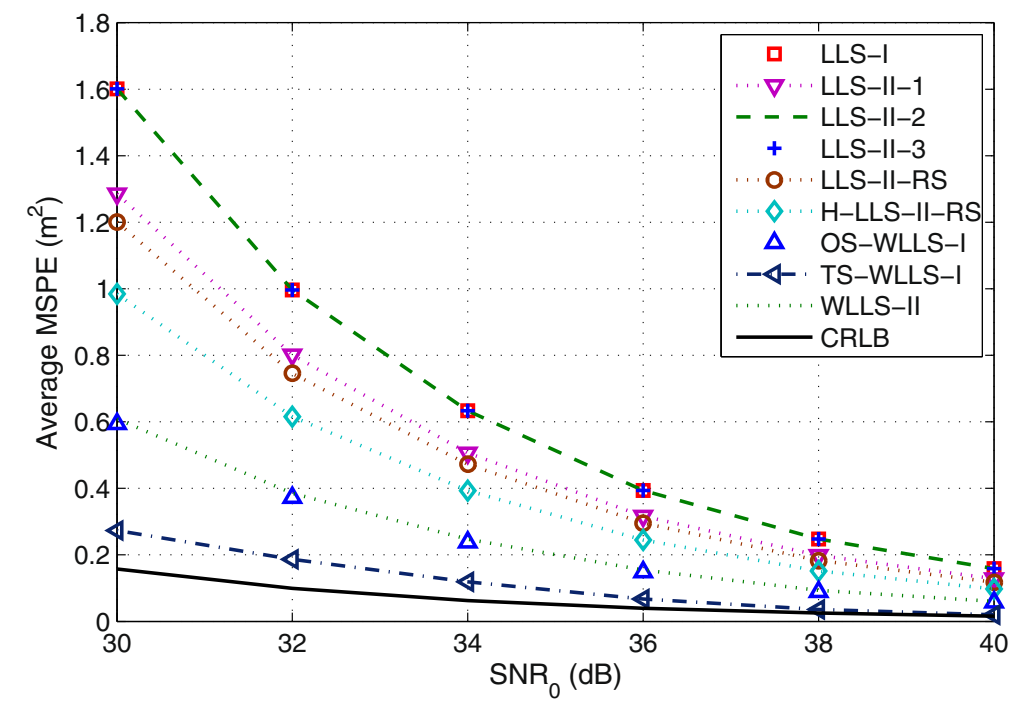

Figure 4 Comparison of different LLS localization algorithms $(\eta=5)$. 
According to [16], the TS-WLLS-I location estimate can be written as

$$
\widehat{\mathbf{p}}_{\text {TS-WLLS-I }}=\left[\operatorname{sgn}\left(\left[\widehat{\Lambda}_{\text {WLLS }}\right]_{1}\right) \sqrt{\left[\widehat{\mathbf{z}}_{1}\right.} \operatorname{sgn}\left([\widehat{\Lambda} \mathrm{WLLS}]_{2}\right) \sqrt{\left[\widehat{\mathbf{z}}_{2}\right]} T\right.
$$

where $\operatorname{sgn}(\cdot)$ represents the signum function,

$$
\begin{aligned}
& \widehat{\mathbf{z}}=\left(\mathbf{G}^{T} \boldsymbol{\Phi}^{-1} \mathbf{G}\right)^{-1} \mathbf{G}^{T} \boldsymbol{\Phi}^{-1} \mathbf{h}, \\
& \mathbf{G}=\left[\begin{array}{ll}
1 & 0 \\
0 & 1 \\
1 & 1
\end{array}\right], \\
& \mathbf{\Phi}=\mathbf{K}\left(\mathbf{A}_{\mathbf{I}}^{T} \mathbf{C}_{\mathbf{I}}^{-1} \mathbf{A}_{\mathbf{I}}\right)^{-1} \mathbf{K}, \\
& \mathbf{K}=\operatorname{diag}\left(2 \times\left[\widehat{\boldsymbol{\Lambda}}_{\mathrm{WLLS}}\right]_{1}, 2 \times\left[\widehat{\boldsymbol{\Lambda}}_{\mathrm{WLLS}}\right]_{2}, 1\right), \\
& \mathbf{h}=\left[\left[\widehat{\boldsymbol{\Lambda}}_{\mathrm{WLLS}}\right]_{1}^{2}\left[\widehat{\boldsymbol{\Lambda}}_{\mathrm{WLLS}}\right]_{2}^{2}\left[\widehat{\boldsymbol{\Lambda}}_{\mathrm{WLLS}}\right]_{3}\right]^{T} .
\end{aligned}
$$

\subsection{WLLS-II localization algorithm}

According to [13], the reference selection has no effect on the WLLS-II localization performance and the WLLSII location estimate can be written as

$$
\widehat{\mathbf{p}}_{\mathrm{WLLS}-\mathrm{II}}=\left(\mathbf{A}_{\text {II }}^{T} \mathbf{C}_{\mathrm{II}}^{-1} \mathbf{A}_{\mathrm{II}}\right)^{-1} \mathbf{A}_{\text {II }}^{T} \mathbf{C}_{\text {II }}^{-1} \mathbf{b}_{\text {II }},
$$

where $\mathbf{C}_{\mathrm{II}}$ is the weighted matrix and its elements can be derived as [13]

$$
\begin{aligned}
{\left[\mathbf{C}_{\mathbf{I I}}\right]_{i j}=} & 4 \hat{d}_{r}^{2} \sigma_{r}^{2}+3 \sigma_{r}^{4}-\sigma_{r}^{2}\left(\sigma_{i}^{2}+\sigma_{j}^{2}\right)+\sigma_{i}^{2} \sigma_{j}^{2}+I(i, j) \\
& \times\left(4 \hat{d}_{i}^{2} \sigma_{i}^{2}+2 \sigma_{i}^{4}\right)
\end{aligned}
$$

with $i, j=1,2, \ldots, N, i \neq r, j \neq r$, and $I(i, j)$ is an indicator function which is 1 for $i=j$, and is 0 otherwise.

\section{Numerical simulation results}

The simulation scenario is depicted in Figure 1, where a hybrid network with $N=4$ anchors is used to localize one agent. The area is a square of $L \times L \mathrm{~m}^{2}$ and $L$ is fixed to $10 \mathrm{~m}$. There are two sets of anchors (set I and set II) in Figure 1. Set I contains $S=2$ TOA-based ranging anchors located at $\mathbf{p}_{1}=(0,0)$ and $\mathbf{p}_{2}=(L, 0)$ respectively. Set II contains N-S $=2$ RSS-based ranging anchors located at $\mathbf{p}_{3}=(L, L)$ and $\mathbf{p}_{4}=(0, L)$, respectively. The agent location $\mathbf{p}$ is changed with $2 \mathrm{~m}$ intervals within [1,9] $\mathrm{m}$ both in $x$ and $y$ directions, yielding a $5 \times 5$ grid of possible agent locations. The mean square position error (MSPE) of different techniques are simulated at each location on the grid, and then average over all the agent locations on the grid. For LLS-II-1, the anchor-1 is selected as the reference anchor.
According to (5) and [8], we simply assume that the agent and anchors have the same configuration and let TOA-based ranging error variance $\sigma_{\text {TOA }, i}^{2}$ be reversely proportional to $\mathrm{SNR}_{i}$, which is

$$
\sigma_{\mathrm{TOA}, i}^{2}=\frac{1}{\mathrm{SNR}_{i}}=\frac{1}{\mathrm{SNR}_{0}}\left(\frac{d_{i}}{d_{0}}\right)^{\gamma}, i \in\{1,2, \cdots, S\}
$$

where $\mathrm{SNR}_{0}$ is the SNR at the reference distance $d_{0}$ and $\gamma$ is the path-loss exponent. According to (8) and [24], we simply assume RSS-based ranging error variance $\sigma_{\mathrm{RSS}, i}^{2}$ as

$$
\sigma_{\mathrm{RSS}, i}^{2}=\eta^{2} \sigma_{\mathrm{TOA}, i}^{2}
$$

where $\eta(\eta \geq 1)$ controls the relation between the two ranging error variances since TOA-based ranging usually has higher accuracy than RSS-based ranging, especially when adopting UWB signals. In the following simulations, we assume $d_{0}=1, \mathrm{SNR}_{0} \in[20: 30] \mathrm{dB}$ with increasing step $\Delta=2 \mathrm{~dB}$, and $\gamma=2$ unless otherwise specified. For each simulation setting, $10^{3}$ simulations are run to get the average performance.

For the non-hybrid TOA-based localization scenario or the hybrid TOA/RSS localization scenario that the variances of both RRS-based and TOA-based ranging are the same, i.e., $\eta=1$, simulation results are depicted in Figure 2. The LLS-II-1 algorithm performs the worst among all the algorithms and the CRLB provides the lower bound for them. The localization accuracy of the LLS-I algorithm is the same as that of both LLS-II-2 and LLS-II-3 algorithms, which is slightly lower than the accuracy of the H-LLS-II-RS algorithm. The LLS-RS algorithm beats all the other LLS algorithms. The reason is that LLS-I, LLS-II-2, and LLS-II-3 algorithms equally utilize all the range measurements (also their errors), while $H$-LLS-II-RS and LLS-II-RS algorithms suppress the influence of ranging errors on the localization accuracy through reference selection. For the non-hybrid TOA-based localization scenario, it is obvious that $H$ LLS-II-RS and LLS-II-RS algorithms are the same, thus having the same performance. However, for the hybrid TOA/RSS localization scenario that the variances of both RRS-based and TOA-based ranging are the same, the LLS-II-RS algorithm outperforms the H-LLS-II-RS algorithm, since the $H-L L S-I I-R S$ algorithm never selects a RSS-based anchor as the reference even if its range measurement is the shortest. Moreover, the WLLS algorithms outperform the LLS algorithms since all measurements are weighted according to their reliability. The OS-WLLS-I algorithm has almost identical localization accuracy to the WLLS-II algorithm, since they both utilize the variances of the measurements. Furthermore, the TS-WLLS-I algorithm beats all the other WLLS algorithms and attains the 
CRLB in high SNR regions, since it further exploit the constraint of the dummy variable.

For the hybrid TOA/RSS localization scenario, if the RSS-based ranging variances are slightly larger than the TOA-based ranging variances, e.g., $\eta=2$, simulation results are depicted in Figure 3. Different from Figure 2, the LLS-II-1 algorithm outperforms LLS-I, LLS-II-2, and LLS-II-3 algorithms. The reason is that the LLS-II-1 algorithm selects a TOA-based anchor with small ranging variance as the reference, which can more effectively suppress the ranging errors on the localization accuracy than those three LLS algorithms, since they equally utilize the inaccurate RSS-based range measurements and the accurate TOA-based range measurements. Moreover, LLS-II-RS and H-LLS-II-RS algorithms have nearly identical localization accuracy, since the effect of larger RSS-based ranging variances plays an almost identical role as that of range measurements on the final localization performance.

For the hybrid TOA/RSS localization scenario, if the RSS-based ranging variances are considerably larger than the TOA-based ranging variances, e.g., $\eta=5$, simulation results are depicted in Figure 4. Since the variances of RSS-based ranging become larger, the $\mathrm{SNR}_{0}$ range is changed to [30:40] $\mathrm{dB}$ to get reasonable localization results. The localization performances of both $L L S-I I-1$ and $L L S$ II-RS techniques are nearly identical and the H-LLS-II-RS technique beats all the other LLS techniques, since the effect of larger RSS-based ranging variances plays a more important role than that of range measurements on the final localization performance.

\section{Conclusions}

If the variances of range measurements are unavailable, the LLS-II localization algorithm should be adopted in both non-hybrid and hybrid networks using their respective reference selection criterions. If the variances of range measurements are available, the two-step WLLS-I algorithm should be utilized to localize the agent in both non-hybrid and hybrid networks. Although only LOS scenarios are considered in the paper, the idea can be extended to NLOS scenarios.

\section{Competing interests}

The authors declare that he has no competing interests.

\section{Acknowledgements}

This research was supported by the Doctor Foundation of Tianjin Normal University (52XB1417).

Received: 2 February 2015 Accepted: 16 February 2015

Published online: 05 March 2015

\section{References}

1. Q Liang, B Zhang, C Zhao, Y Pi, TDoA for passive localization: underwater versus terrestrial environment. IEEE Trans Parallel Distributed Syst 24(10), 2100-2108 (2013)
2. Q Liang, L Wang, Q Ren, Fault tolerant and energy efficient cross-layer design for wireless sensor networks. Int I Sensor Netw 2(3/4), 248-257 (2007)

3. H Shu, Q Liang, Fuzzy optimization for distributed sensor deployment, 3rd edn. (Proc. IEEE Wireless Communications and Networking Conference (WCNC), New Orleans, LA, 2005), pp. 1903-1908

4. Q Liang, Waveform design and diversity in radar sensor networks: theoretical analysis and application to automatic target recognition, 2nd edn. (Proc. Third Annual IEEE Communications Society Conference on Sensor, Mesh and Ad Hoc Communications And Networks (SECON), Reston, VA, 2006), pp. 684-689

5. E Jain, Q Liang, Sensor placement and lifetime of wireless sensor networks: theory and performance analysis, 1st edn. (Proc. IEEE Globe Telecommunications Conference (GLOBECOM, St. Louis, MO, 2005), pp. 173-177

6. SA Zekavat, RM Buehrer, Handbook of position location: theory, practice, and advances (John Wiley \& Sons, NJ, 2011)

7. D Dardari, M Luise, E Falletti, Satellite and terrestrial radio positioning techniques: a signal processing perspective (Elsevier, Oxford, 2012)

8. S Gezici, HV Poor, Position estimation via ultra-wide-band signals. Proc IEEE 97(2), 386-403 (2009)

9. D Dardari, A Conti, U Ferner, A Giorgetti, MZ Win, Ranging with ultrawide bandwidth signals in multipath environments. Proc IEEE 97(2), 404-426 (2009)

10. JC Chen, RE Hudson, K Yao, Maximum-likelihood source localization and unknown sensor location estimation for wideband signals in the near field. IEEE Trans Signal Process 50(8), 1843-1854 (2002)

11. JJ Caffery, A new approach to the geometry of TOA location, 4th edn. (Proc. IEEE Vehicular Technology Conference (VTC), Boston, 2000), pp. 1943-1949

12. I Guvenc, S Gezici, F Watanabe, H Inamura, Enhancements to linear least squares localization through reference selection and ML estimation (Proc. IEEE Wireless Communications and Networking Conference (WCNC), Las Vegas, 2008), pp. 284-289

13. Y Wang, $\mathrm{F}$ Zheng, M Wiemeler, W Xiong, T Kaiser, Reference selection for hybrid TOA/RSS linear least squares localization (Las Vegas, Proc. IEEE Vehicular Technology Conference (VTC), 2013), pp. 1-5

14. S Venkatesh, RM Buehrer, A linear programming approach to NLOS error mitigation in sensor networks (Proc. International Conference on Information Processing in Sensor Networks (IPSN), Nashville, TN, 2006), pp. 301-308

15. Z Li, W Trappe, Y Zhang, B Nath, Robust statistical methods for securing wireless localization in sensor networks (Proc. International Symposium on Information Processing in Sensor Networks (IPSN), LA, 2005), pp. 91-98

16. YT Chan, $\mathrm{KC} \mathrm{Ho}$, A simple and efficient estimator for hyperbolic location. IEEE Trans Signal Process 42(8), 1905-1915 (1994)

17. KW Cheung, W-K Ma, H So, YT Chan, Least squares algorithms for time-of-arrival based mobile location. IEEE Trans Signal Process 52(4), 1121-1128 (2004)

18. KW Cheung, W-K Ma, H So, YT Chan, A constrained least squares approach to mobile positioning: algorithms and optimality. EURASIP J. Adv. Signal Process 2006, 1-23 (2006)

19. A Catovic, Z Sahinoglu, The Cramer-Rao bounds for hybrid TOA/RSS and TDOA/RSS location estimation schemes. IEEE Commun Lett 8(10), 626-628 (2004)

20. M Laaraiedh, S Avrillon, B Uguen, Cramer-Rao lower bounds for nonhybrid and hybrid localization techniques in wireless networks. Trans Emerg Telecommun Technol 23(3), 268-280 (2012)

21. Y Wang, M Wiemeler, F Zheng, W Xiong, T Kaiser, Two-step hybrid self-localization using unsynchronized low-complexity anchors (Proc. International Conference on Localization and GNSS (ICL-GNSS), Turin, Italy, 2013), pp. 1-5

22. Y Shen, MZ Win, Fundamental limits of wideband localization part I: a general framework. IEEE Trans Inf Theory 56(10), 4956-4980 (2010)

23. AF Molisch, D Cassioli, C-C Chong, S Emami, A Fort, B Kannan, J Karedal, J Kunisch, HG Schantz, K Siwiak, MZ Win, A comprehensive standardized model for ultrawideband propagation channels. IEEE Trans Antennas Propagation 54(11), 3151-3166 (2006)

24. T Gadeke, J Schmid, M Kruger, J Jany, W Stork, KD Muller-Glaser, A bi-modal ad-hoc localization scheme for wireless networks based on RSS and ToF fusion (Proc Workshop on Positioning, Navigation and Communication (WPNC), Dresden, Germany, 2013), pp. 1-6 